\title{
NC Algorithms for Computing a Perfect Matching, the Number of Perfect Matchings, and a Maximum Flow in One-Crossing-Minor-Free Graphs
}

\author{
David Eppstein \\ Vijay V. Vazirani \\ eppstein@uci.edu \\ vazirani@ics.uci.edu \\ Computer Science Department, University of California, Irvine \\ Irvine, California
}

\begin{abstract}
In 1988, Vazirani gave an NC algorithm for computing the number of perfect matchings in $K_{3,3}$-minor-free graphs by building on Kasteleyn's scheme for planar graphs, and stated that this "opens up the possibility of obtaining an NC algorithm for finding a perfect matching in $K_{3,3}$-free graphs." In this paper, we finally settle this 30 year-old open problem. Building on recent NC algorithms for planar and bounded-genus perfect matching by Anari and Vazirani and by Sankowski, we obtain NC algorithms for perfect matching in any minor-closed graph family that forbids a one-crossing graph. This result applies to several well-studied graph families including the $K_{3,3}$-minor-free graphs and $K_{5}$-minor-free graphs. Graphs in these families not only have unbounded genus, but can have genus as high as $O(n)$. Our method applies as well to several other problems related to perfect matching. In particular, we obtain NC algorithms for the following problems in any family of graphs (or networks) with a one-crossing forbidden minor:
\end{abstract}

- Determining whether a given graph has a perfect matching and if so, finding one.

- Finding a minimum weight perfect matching in the graph, assuming that the edge weights are polynomially bounded.

- Computing the number of perfect matchings in the graph.

- Finding a maximum $s t$-flow in the network, with arbitrary capacities.

The main new idea enabling our results is the definition and use of matching-mimicking networks, small replacement networks that behave the same, with respect to matching problems involving a fixed set of terminals, as the larger network they replace.

\section{ACM Reference Format:}

David Eppstein and Vijay V. Vazirani. 2019. NC Algorithms for Computing a Perfect Matching, the Number of Perfect Matchings, and a Maximum Flow in One-Crossing-Minor-Free Graphs. In 31st ACM Symposium on Parallelism in Algorithms and Architectures (SPAA '19), fune 22-24, 2019, Phoenix, AZ, USA. ACM, New York, NY, USA, 8 pages. https://doi.org/10.1145/3323165.3323206

Permission to make digital or hard copies of all or part of this work for personal or classroom use is granted without fee provided that copies are not made or distributed for profit or commercial advantage and that copies bear this notice and the full citation on the first page. Copyrights for components of this work owned by others than the author(s) must be honored. Abstracting with credit is permitted. To copy otherwise, or republish, to post on servers or to redistribute to lists, requires prior specific permission and/or a fee. Request permissions from permissions@acm.org.

SPAA '19, fune 22-24, 2019, Phoenix, AZ, USA

(c) 2019 Copyright held by the owner/author(s). Publication rights licensed to ACM. ACM ISBN 978-1-4503-6184-2/19/06 . .\$15.00

https://doi.org/10.1145/3323165.3323206

\section{INTRODUCTION}

Obtaining an NC algorithm for matching has been an outstanding open question in theoretical computer science for over three decades, ever since the discovery of RNC matching algorithms $[24,32]$. In a recent breakthrough result, Anari and Vazirani gave an NC algorithm for finding a perfect matching in planar graphs [1]. Subsequently, Sankowski provided an alternative algorithm for the same problem based on different techniques [35]. By using a reduction from flow problems on other surfaces to planar flow [4], Anari and Vazirani also extended their result to graphs of bounded genus. Their paper restated the open problem of obtaining an NC algorithm for finding a perfect matching in $K_{3,3}$-minor-free graphs, in particular because such graphs can have genus as high as $O(n)$. This problem was previously stated by Vazirani in a 1989 paper in which he gave an NC algorithm for computing the number of perfect matchings in such graphs [39] and stated that this "opens up the possibility of obtaining an $\mathrm{NC}$ algorithm for finding a perfect matching in $K_{3,3}$-free graphs." In this paper, we finally settle this 30-year-old open problem.

The $K_{3,3}$-minor-free graphs are particularly attractive as a target for this problem because they form a natural extreme case for certain approaches. In particular, they are known to have Pfaffian orientations, by which their matchings can be counted using matrix determinants $[25,28]$, while for $K_{3,3}$ itself and for any minor-free family that does not forbid it, this tool is unavailable. However, our result breaks through this barrier: we give an NC algorithm for finding a perfect matching in graphs belonging to any onecrossing-minor-free class of graphs. That is, if $H$ is any graph that can be drawn in the plane with only one crossing pair of edges, then we can find perfect matchings in the $H$-minor-free graphs in NC. Because $K_{3,3}$ can be drawn with one crossing (Figure 2), our result includes in particular the $K_{3,3}$-minor-free graphs.

In the full version of this paper, we build on these ideas to give NC algorithms for computing, in the same families of graphs, a perfect matching of minimum weight when the weights are polynomiallybounded integers, and the number of perfect matchings.

\footnotetext{
${ }^{1}$ Vazirani stated this problem in terms of graphs with no subgraph homeomorphic to $K_{3,3}$, rather than with no $K_{3,3}$ minor. However, for 3-regular graphs such as $K_{3,3}$, subgraphs homeomorphic to $H$ are equivalent to $H$-minors. We use the formalism of minors because it fits better with our generalization to other forbidden minors. We vary from Vazirani in using the terminology " $K_{3,3}$-minor-free" rather than " $K_{3,3}$-free" to avoid confusion with a third, unrelated meaning, the graphs that do not have $K_{3,3}$ as induced subgraphs.
} 
In another direction, we obtain an NC algorithm for finding a maximum $s t$-flow in any flow network whose underlying undirected graph belongs to a one-crossing-minor-free family. This generalizes Johnson's 1987 result [22], showing that maximum stflow in a planar network is in NC. Again, we defer the details to the full version.

\subsection{Technical ideas}

Our main new technical idea is that of a matching-mimicking network. Given a graph $G$ and a set $T$ of terminal vertices, a matchingmimicking network is a graph $G^{\prime}$, containing $T$, that has the same pattern of matchings: every matching of $G$ that covers $G \backslash T$ corresponds to a matching of $G^{\prime}$ that covers $G^{\prime} \backslash T$ and vice versa.

We show that matching-mimicking networks exist for graphs with any bounded number of terminals. The size of these networks is bounded by a function of the number of terminals. For at most three terminals, we describe these networks explicitly. In this case, the mimicking networks are planar and remain planar when glued into the triangular face of a larger planar network, and can be given edge weights so that their minimum-weight perfect matchings (for each subset of terminals) mimic the weights of the minimumweight matchings in the given graph. Both of these properties, their planarity and their weight-mimicking ability, are needed by our algorithm.

In the past, mimicking networks for network flow were defined and used by numerous researchers for obtaining flow algorithms [6, 7, 19, 26, 27]. These mimicking networks were first defined to prove that maximum flow can be found in NC in graphs of bounded treewidth [19] and later used also in efficient sequential algorithms for flow in one-crossing-minor-free graphs [6]. Their theoretical properties have also become an object of study in their own right $[7,26,27]$. It seems likely that, similarly, our matchingmimicking networks will lead to algorithmic applications beyond our NC matching algorithm, and additional theory beyond our existence proof.

As with a previous sequential flow algorithm of Chambers and Eppstein [6], we exploit the structural decomposition of graphs with a one-crossing forbidden minor [34], by repeatedly using mimicking networks to simplify this structure. However the order in which we perform these replacements must be more carefully chosen so that our algorithms run in NC. Each step of the replacement process involves the computation of matchings either in a boundedtreewidth graph or in a planar graph. The planar matchings can be found by the new results of Anari and Vazirani or of Sankowski, and the bounded-treewidth matchings can be found in NC by using log-space versions of Courcelle's theorem [11]. ${ }^{2}$

Our maximum st-flow result uses a similar algorithmic outline, with flow-mimicking networks in place of matching-mimicking networks. Our method differs from the sequential algorithm of Chambers and Eppstein [6], which used flow-mimicking networks on at most three terminals to replace leaf nodes of the structural

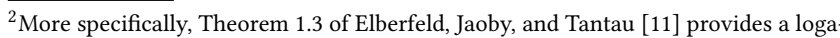
rithmic space algorithm, for any monadic second order graph property with a free set variable, that counts the sets of each cardinality for which a given bounded-treewidth graph models the formula. Matching may be expressed in this way: a set $S$ of edges forms a matching if the graph models a formula stating that no two edges of $S$ share a vertex. The claim follows from the inclusion of LOGSPACE in NC.
}

decomposition tree. Our matching algorithms, also, use matchingmimicking networks on at most three terminals, replacing subtrees of more than one node in a single step. However, our parallel flow algorithm uses flow-mimicking networks for a second purpose, namely to replace a component of our matching algorithm that involves semiring matrix multiplication. This part of our flow algorithm requires flow-mimicking networks on up to six terminals.

\subsection{History and related results}

The first result on the problem of obtaining a fast parallel algorithm for matching was obtained by Lovasz [29]. In a seminal paper, he proposed a way of computing a perfect matching via methods quite different from the combinatorial (augmenting-path-finding) methods that were the mainstay at the time. Using the Tutte matrix of the graph, he proposed methods from linear algebra and randomization.

When combinatorial methods were found to be lacking for obtaining a fast parallel matching algorithm, researchers turned to Lovasz's proposed method. The first RNC algorithm for finding a perfect matching was obtained by Karp, Upfal, and Wigderson [24]. This was followed by a somewhat simpler algorithm due to Mulmuley, Vazirani, and Vazirani [32]. Matching has played a central role in the development of the theory of algorithms, in that its study, from various computational viewpoints, has led to quintessential paradigms and powerful tools for the entire theory. The two parallel matching algorithms also led to such gains: the first led to a fundamental understanding of the computational relationship between search and decision problems [23] and the second yielded a basic probabilistic technique encapsulated as the Isolation Lemma [32], which has found several applications in complexity theory and algorithms.

However, this still did not clarify whether randomization was essential for fast parallel matching. Considering the fundamental insights gained by an algorithmic study of matching, this has remained a significant open question ever since the 1980s. The first substantial progress on this question was made by Miller and Naor in 1989 [31]. They obtained an NC algorithm for finding a maximum flow from a set of sources to a set of sinks in a planar network; as a corollary, they obtained an NC algorithm for finding a perfect matching in bipartite planar graphs. As is well known, Kasteleyn's algorithm for counting the number of perfect matchings in a planar graph [25] can be easily made into an NC algorithm for counting perfect matchings by using Csanky's NC algorithm for the determinant of a matrix [8]. In 2000, Mahajan and Varadarajan gave an elegant way of using this NC algorithm for counting perfect matchings to find one, hence giving a different $\mathrm{NC}$ algorithm for bipartite planar graphs [30]. (The NC counting algorithm was also used critically in Anari and Vazirani's NC algorithm for non-bipartite planar graphs [1].)

After a decade and half of lull, there has been a resurgence of activity on this problem in the last couple of years. In particular, several researchers have obtained quasi-NC algorithms for matching and its generalizations. Such an algorithm runs in polylogarithmic time; however, it requires $O\left(n^{\log O(1)} n\right)$ processors. All the algorithms in this line of research work by a partial derandomization of the Isolation Lemma. This line of work was started by Fenner, 


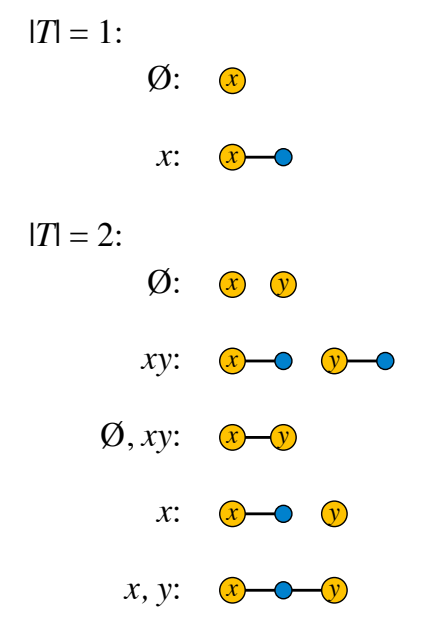

$$
|T|=3 \text { : }
$$

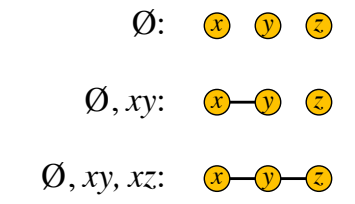

$\emptyset, x y, x z, y z:$
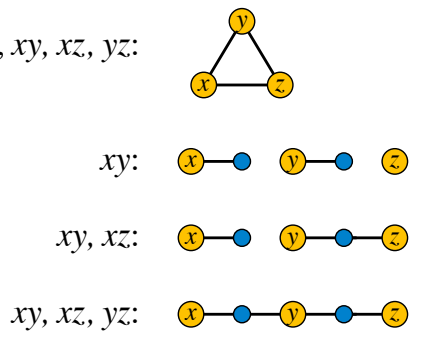

$x: \stackrel{\longrightarrow}{\longrightarrow}$ (1) (2)

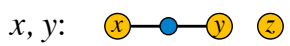

$x, y, z:$ (y)

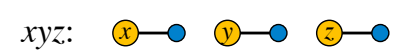

$x y z, x: \stackrel{(x)}{\longrightarrow}$ (1)-2)

$x y z, x, y:$ (x)-(y)-2

$x y z, x, y, z$ :

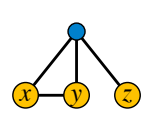

Figure 1: Matching-mimicking networks for $|T| \leq 3$. In each network, the set $T$ of terminals consists of the labeled yellow vertices; the remaining non-terminal vertices of the network are the smaller blue vertices. The labels denote the subsets of terminals that can be covered by a matching.

Gurjar, and Thierauf, who gave a quasi-NC algorithm for perfect matching in bipartite graphs [14]. Later, Svensson and Tarnawski extended the result to general graphs [37]. The generalization of bipartite matching to the linear matroid intersection problem was given by Gurjar and Thierauf [17] and to finding a vertex of a polytope with totally unimodular constraints by Gurjar, Thierauf, and Vishnoi [18]. Researchers have also developed pseudo-deterministic RNC matching algorithms, which output the same (unique) solution for almost all choices of random bits $[2,16]$.

The first NC algorithm for finding a maximum $s t$-flow in a planar network was obtained by Johnson [22]. As stated above, this was followed by an $\mathrm{NC}$ algorithm for finding a maximum flow from a set of sources to a set of sinks in a planar network by Miller and Naor [31]. An NC algorithm for maximum flow in graphs of bounded treewidth was given by Hagerup et al. [19].

\section{MATCHING-MIMICKING NETWORKS}

A matching, in an undirected graph, is a subset of edges no two of which share a vertex. The matching covers a subset of vertices, the ones that are vertices of the selected edges. If $G$ is a graph with a specified subset $T$ of vertices, we define the matching pattern of $G$ to be a family of subsets of $T$, the subsets $X \subset T$ such that some matching of $G$ covers $(G \backslash T) \cup X$ (and covers no other vertices). If $G$ and $G^{\prime}$ are two graphs, both containing a shared subset $T$ of vertices, we say that $G$ and $G^{\prime}$ are matching-equivalent on $T$ if they have the same matching patterns. A matching-mimicking network for $G$ and $T$ is any other graph $G^{\prime}$ containing $T$ that is matching-equivalent to $G$ on $T$.

LEMMA 1. There is a function $f$ such that any graph $G$ and subset of vertices $T$ has a matching-mimicking network of at most $f(|T|)$ vertices.

Proof. This follows immediately from the fact that $T$ has $2^{|T|}$ subsets, and therefore that $G$ has at most $2^{2^{|T|}}$ matching patterns. For each matching pattern that can be achieved, we may take the mimicking network to be the smallest graph that achieves that matching pattern. The worst-case size of the resulting mimicking network is the largest size of a finite set of graphs (one for each achievable matching pattern) and is therefore bounded as a function of $|T|$.

We have performed by hand the case analysis needed to construct matching-mimicking networks for $|T| \leq 3$, the largest number of terminals needed for our algorithms. The results are depicted in Figure 1. As the figure shows, for $|T|=3$ the number of matching patterns is 14 , much smaller than the $2^{2^{3}}=256$ bound on the number of patterns obtained by plugging $|T|=3$ into the proof of Lemma 1. We achieve this reduction in the number of cases by combining the following three observations:

- We omit graphs whose matching pattern is empty. If any such graph is detected during our algorithm for matching, we may abort the algorithm, as the whole graph has no matching.

- The sizes of the subsets of $T$ in any single matching pattern must all have the same parity as each other.

- We may consider matching patterns to be equivalent whenever one matching pattern can be obtained from another by permuting the vertices of $T$. We only need to find matchingmimicking networks for each equivalence class of matching patterns.

The following property indicates that, when glued into a planar graph, all of the matching-mimicking networks of the figure preserve its planarity. We need this property in our algorithm, so that we can continue to compute matchings in the result of such gluings.

Lemma 2. Let $G$ be a planar graph, let $f$ be a triangular face of a planar drawing of $G$, and let $T$ be a subset of the vertices of $f$. Then the union of $G$ and any of the matching-mimicking networks of Figure 1, with terminal set $T$, is another planar graph that can be drawn in the plane with the matching-mimicking network inside $f$. 

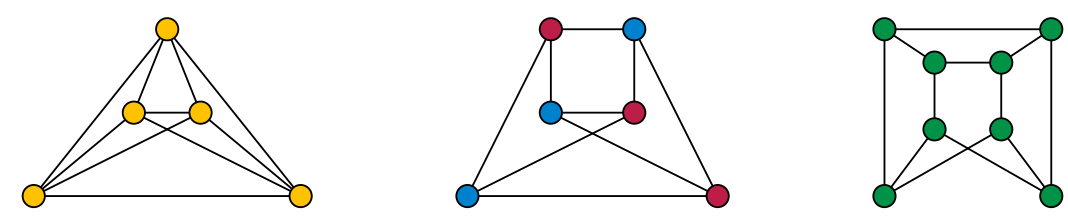

Figure 2: $K_{5}$ (left), $K_{3,3}$ (center), and the eight-vertex Wagner graph (right) are all nonplanar but can all be drawn in the plane with only one crossing.

Proof. This follows from the layouts given for these networks in the figure, which are all drawn outerplanar (planar and with all vertices belonging to the unbounded face of the drawing). Because they are outerplanar, their unbounded face can be surrounded by triangle $f$ and then, around $f$, the rest of $G$, without creating any new crossings.

\section{STRUCTURAL DECOMPOSITION}

A $k$-clique-sum of two graphs is defined as a graph that can be obtained from the disjoint union of the two given graphs by identifying a clique of $\leq k$ vertices in one of the graphs with a clique of the same size in the other graph, and then optionally deleting some of the edges of the merged clique. One-crossing-minor-free graphs have a structural decomposition that can be described in terms of clique-sums: If $H$ is a graph that can be drawn in the plane with at most one edge crossing, then the $H$-minor-free graphs can be decomposed by 3-clique-sums into pieces that are either planar or of bounded treewidth [34]. This decomposition generalizes the result that graphs with a planar forbidden minor have bounded treewidth, and is a simplified form of the structural decomposition of arbitrary minor-closed graph families by Robertson and Seymour, which also includes pieces of bounded genus, apexes (vertices that can be adjacent to any subset of the other vertices in a single piece), and vortexes (subgraphs of bounded pathwidth attached to a face of a bounded-genus piece). The graphs with one-crossing drawings include $K_{3,3}$ and $K_{5}$ (Figure 2), whose corresponding minor-free graph classes have even simpler forms of this decomposition: the $K_{3,3}$-minor-free graphs are 2-clique-sums of planar graphs and $K_{5}$, and the $K_{5}$-minor-free graphs are 3-clique-sums of planar graphs and the eight-vertex Wagner graph (shown in Figure 2, right).

We can describe any clique-sum decomposition, such as the decomposition from this structure theorem, as a two-colored tree (Figure 3), in which the nodes of one color represent pieces (planar graphs or bounded-treewidth graphs), and the nodes of the other color represent cliques on which two are more pieces are glued. The edges of this tree describe the incidence relation between edges and cliques. Additionally, each clique node of the decomposition tree is labeled with information describing which of its edges are kept as part of the overall graph. When a planar piece is glued to other graphs along a non-facial triangle, we may split the planar piece into two smaller pieces on that triangle. Therefore, it is safe to assume that, for each planar piece of the decomposition, the 3-vertex cliques incident to it are all faces of a planar embedding of the piece.

For our algorithms, it is necessary not merely to know that this 3clique-sum decomposition exists, but also to find it, in NC. Efficient decomposition algorithms are known for $K_{3,3}$-minor-free graphs and for $K_{5}$-minor-free graphs [3,33], but they are sequential, and we are not aware of such algorithms for the general case. In previous work on sequential flow algorithms we avoided this issue by assuming that the decomposition was given as part of the input [6]. Fortunately, in our new results, we do not need the decomposition to be efficient; it merely needs to be in NC.

A subset of three vertices is a separator if its removal would increase the number of connected components of the remaining graph. We are interested in finding a decomposition of a given graph, assumed to be from a one-crossing-minor-free family, by minimal separators of at most three vertices. However, this decomposition is not unique; for instance $K_{3,3}$ has two incompatible minimal separators, the two sides of its bipartition. We define a family of separators to be laminar when no two vertices from any one separator in the family are separated by any other separator in the family. There exist graphs (such as the wheel graph) for which there are quadratically many separating triples, most pairs of which are non-laminar, but maximal laminar sets of separating triples in planar graphs may be found sequentially in linear time [13]. Our graphs are non-planar, and again we are only interested in membership in NC.

LEMMA 3. We can find the structural decomposition of graphs in any one-crossing-minor-free family, as described above, in NC.

We defer the details to the full version of this paper. Additionally we can check which pieces are planar, and find a planar embedding for the planar pieces, in NC [21].

It will be convenient to define one more tool, a structural decomposition of our structural decomposition. It is the heavy path decomposition of a tree (the tree describing the structural decomposition). If any tree $T$ is given an arbitrary root, it may be decomposed into paths by choosing at each non-leaf node of the tree a single child, the one with the most descendants (counting each node as one of its own descendants, and choosing arbitrarily in case of ties). The chosen parent-child edges link together to form a cover of $T$ by vertex-disjoint paths, including some length-zero paths for unchosen leaf vertices. These paths are the heavy paths of the decomposition. Each heavy path (other than the one containing the root vertex) has a parent path, the path containing the parent of the topmost vertex in the path. We may define the rank of a heavy path whose root has $k$ descendants to be $\left\lfloor\log _{2} k\right\rfloor$. An example is shown in Figure 4.

Then in a heavy path decomposition of a tree with $n$ nodes, all ranks are integers in the range from 0 to $\left\lfloor\log _{2} n\right\rfloor$. If $P$ is any path that does not contain the root, the rank of $P$ is strictly less than the rank of the parent of $P$, because $P$ must have at most half as many descendants as its parent (if it had more, it would have been 

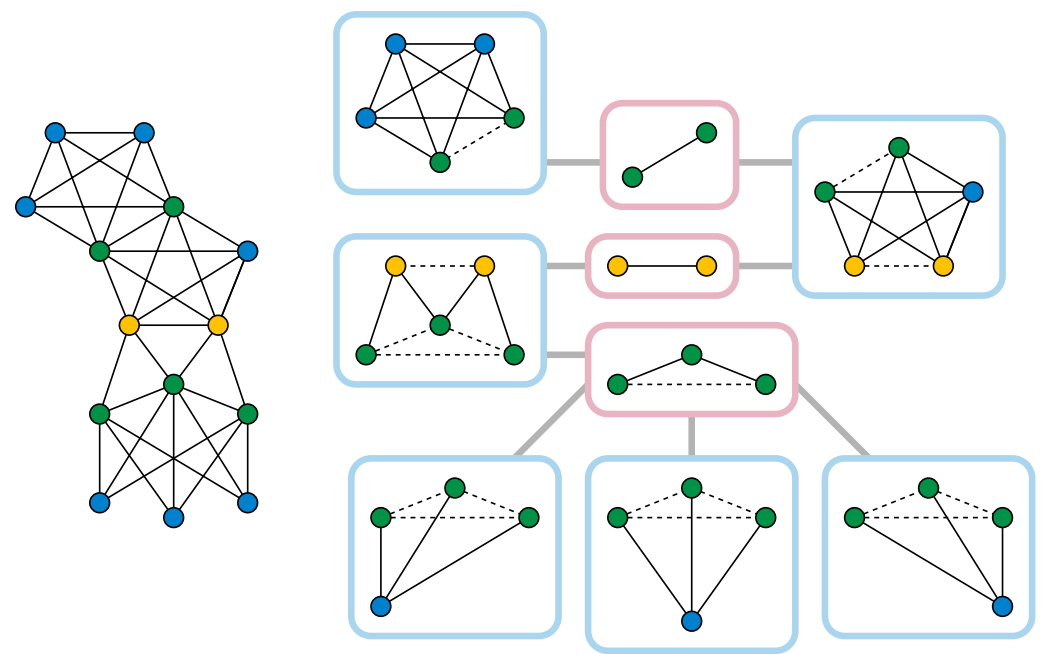

Figure 3: Decomposition of a graph into a 3-clique-sum of simpler pieces (light blue outline) and cliques (light red outline). The dashed edges indicate edges of glued cliques that are to be removed after gluing, either because they are redundant (the same edge appears elsewhere) or because they are not part of the original graph.

picked as the heavy child from the parent of the top node of $P$ ). The heavy path decomposition was introduced for its applications in sequential data structures $[20,36]$, and has become a standard tool for graph drawing and geometric graph algorithms [10, 12], but it has also been recently applied in parallel algorithms [15]. By using the Euler tour technique for trees [38], we may easily count the descendants of each node in a tree, obtaining the following result.

LEMMA 4. We may find the heavy path decomposition of any tree, together with the ranks of each of its paths, in NC.

\section{PERFECT MATCHING ALGORITHM}

To find a perfect matching in a given graph $G$, from a one-crossingminor-free family $\mathcal{F}$, we perform the following steps.

(1) We apply Lemma 3 to find a decomposition of $G$ into a 3clique-sum of pieces that are labeled as either planar or of bounded treewidth.

(2) We root the decomposition tree arbitrarily, and use Lemma 4 to find a heavy path decomposition of the resulting rooted tree structure.

(3) For each rank $r$ from 0 to $\left\lfloor\log _{2} n\right\rfloor$ of a path in the heavy path decomposition (sequentially), we perform the following steps.

(a) In parallel, for each heavy path $P$ of rank $r$, we replace $P$ in the structural decomposition of $G$ by a single matching mimicking network. The terminals of this mimicking network are the (at most three) vertices of $G$ by which $P$ attaches to its parent in the structure tree, and the graph it mimics is the one formed by the clique-sum of all pieces of the decomposition of $G$ that either belong to $P$ or descend from $P$. (We will describe how to construct this mimicking network below.)

(b) We define a shallow clique of the decomposition to be a node of the rooted decomposition tree, representing a clique at which two or more pieces are attached to each other, such that all child pieces (including the one on the heavy path through the node) are mimicking networks (rather than larger pieces or subtrees), and such that the parent piece is planar. At each shallow clique, in parallel, we replace the clique-sum of the attached mimicking networks (a graph of bounded treewidth) with a single mimicking network for the clique-sum, and then replace the parent piece with its clique-sum with this mimicking network, removing the shallow clique and its descendants from the decomposition tree. By Lemma 2 this operation preserves the planarity of the parent piece. Because we remove the clique from the decomposition tree, it also preserves the property that in planar pieces of the decomposition, all 3-clique-sums occur on face triangles.

(4) We reverse the sequence of replacements by matching-mimicking networks, maintaining throughout the reversed sequence a perfect matching for the current graph. When multiple pieces were replaced in parallel, we perform their reversed replacement in parallel in the same way. To reverse the replacement of a single graph by a mimicking network, given a matching in the mimicking network, we construct a corresponding matching in the graph that was replaced, covering the same terminal vertices and all nonterminal vertices.

It remains to explain how to perform step 3(a), in which we construct the mimicking network for a heavy path, and also how we maintain enough information about how we constructed it to reverse the replacement of the path in step 4 .

LEMMA 5. In the algorithm described above, we can compute a mimicking network for each given heavy path, in NC. Moreover, given 


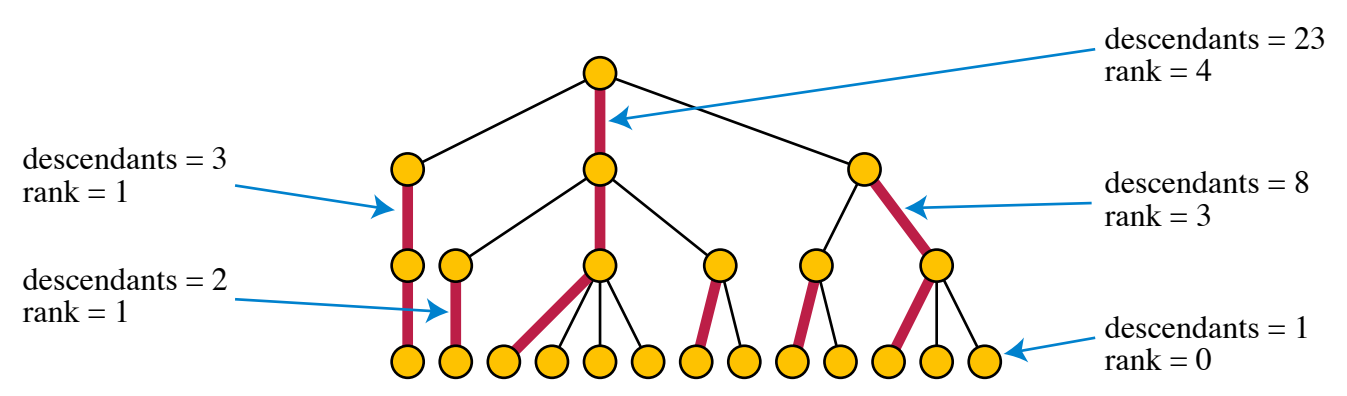

Figure 4: Heavy path decomposition of a rooted tree, showing the number $k$ of descendants of the top node of each path (including itself) and the rank $\left\lfloor\log _{2} k\right\rfloor$ of the path.

a matching in this mimicking network, we can compute a corresponding matching in the clique-sum of the pieces in the path and its descendants, again in NC.

Proof. At the time our algorithm processes a heavy path $P$, all heavy paths of lower rank (and in particular all paths descending from $P$ ) will have already been processed. Therefore, the nodes of $P$ will be of three types, each associated with a subgraph:

- Planar pieces of the decomposition, possibly with glued-in mimicking networks from lower-rank paths that preserve the planarity of the piece. We define the subgraph associated with the node to be this planar piece.

- Bounded-treewidth pieces of the decomposition, possibly attached by clique-sums to mimicking networks from lowerrank paths. We define the subgraph associated with the node to be the clique-sum of it and its attached mimicking networks. Because it is a clique-sum of bounded-treewidth graphs, this associated subgraph has bounded treewidth.

- Cliques of the decomposition, again possibly attached by clique-sums to mimicking networks from lower-rank paths. As with the bounded-treewidth pieces, we define the subgraph associated with the node to be the (bounded-treewidth) clique-sum of this clique with its attached mimicking networks.

For each node interior to $P$, define the two sides of the node to be the two sets of at most three vertices by which its subgraph is connected to its neighbors in $P$. Similarly, for the topmost node of $P$ (the one closest to the root of the decomposition tree) we let one of the sides be the set of at most three vertices connecting it to its parent, and for the bottommost (leaf) node of $P$ we define one of its sides to be the empty set.

Then the matchings that are possible for the subgraph associated with each node can be summarized by a Boolean matrix, the transfer matrix of the node. The rows of the matrix are indexed by subsets of the vertices on one side of the node, the side closest to the leaf of the path, and the columns of the matrix are indexed by subsets of the vertices on the other side. We set the entry of this matrix in row $i$ and column $j$ to be true if there exists a matching of the subgraph associated with the node that covers all interior (non-side) vertices of the subgraph, and covers the subsets of the two sides indexed by $i$ and $j$. Taking into account the fact that, within a single side of each node, the subsets of vertices that can be matched are constrained to all have the same parity as each other, ${ }^{3}$ these matrices have dimension at most $4 \times 4$, but are in some cases smaller. For instance, the transfer matrix of the leaf node of the path has only one row, corresponding to the empty set, as its set of vertices on the leaf side is the empty set. Each Boolean value in each of these transfer matrices can be found in parallel by testing for the existence of a perfect matching in the induced subgraph of the vertices that should be covered.

The transfer matrix for the clique-sum of any contiguous subsequence of nodes in the path is just the product of its matrices, over the Boolean $(\vee, \wedge)$ semiring, in left-to-right order from the leaf end to the root end. As with any product of matrices, we can compute the product matrix in NC, for instance by associating the nodes of the subsequence with the leaves of a balanced binary tree and, at each interior node of the binary tree, multiplying the matrices from the two child nodes. Because the leftmost (leaf) matrix is a row vector, the product of all the matrices will also be a row vector, indicating which subsets of the three vertices on the top side of the top node of $P$ can be covered by a matching that also covers all vertices belonging to the subgraphs associated with $P$. The information in this row vector is exactly what we need to compute a mimicking network for $P$.

To make this process reversible, whenever we compute the product of two transfer matrices we also store, for each true Boolean value in the product matrix, a pair of true Boolean values in the two multiplicands that cause that product value to be true (choosing arbitrarily when multiple pairs would cause it to be true). Then, when we have chosen a matching in the mimicking network for $P$ and wish to replace it by a matching in the subgraphs associated with $P$, we trace back through this stored information to find a sequence of true Boolean values in the transfer matrices of each node of $P$ that together correspond to a matching of the correct type. Then, in each subgraph associated with a node of $P$, we perform a matching algorithm to find a single matching corresponding to the position of this value in its transfer matrix.

THEOREM 6. We can find a perfect matching in any graph from a one-crossing-minor-minimal graph family, in NC.

Proof. All mimicking networks used to replace other pieces in the decomposition tree have bounded size, and therefore bounded

\footnotetext{
${ }^{3}$ The parity of the set of matched vertices on one side of a node must equal the parity of the set of vertices in the input graph minus the node, on the same side. For otherwise, not every vertex in that component could be matched.
} 
treewidth. When we merge a shallow clique into its parent in the decomposition tree, we are gluing a single mimicking network for at most three terminals into a vertex, edge, or triangle of the parent graph. We only perform this merge step once per vertex, edge, or triangle of the parent piece. The parent piece must have been planar before the gluing step, and by Lemma 2 it remains planar. Correspondingly, because this gluing step performs a 3-clique-sum of a graph of bounded treewidth, if the parent piece was of bounded treewidth before the gluing step, it remains of bounded treewidth Thus, all the replacements performed by the algorithm preserve the structure of the decomposition, allowing the algorithm to continue correctly in later steps.

There are logarithmically many iterations of the outer loop, and each iteration performs only steps that can be performed in NC. Therefore, the overall algorithm is also in NC.

\section{DISCUSSION AND OPEN PROBLEMS}

We conclude with some open problems that result from our work:

- Can we prove an explicit upper bound (for instance a closed form formula or primitive recursive function) on the size of matching-mimicking networks, as a function of the number of terminals? Do weighted matching-mimicking networks exist for arbitrary numbers of terminals?

- Because Anari and Vazirani [1] show how to find perfect matchings in bounded-genus graphs, our method immediately extends to facial 3-clique-sums of bounded-genus graphs and bounded-treewidth graphs. However, it is not clear what happens when bounded-genus pieces are glued by clique-sums on triangles that are not faces. Do the facial 3-clique-sums of bounded-genus graphs and boundedtreewidth graphs include any other natural graph classes?

- Is it possible to use the structure theorem for more general minor-closed graph families, allowing non-facial cliquesums, apexes, and vortexes, to find perfect matchings in such families in NC?

- Chambers and Eppstein [6] used mimicking networks to find near-linear-time sequential maximum flow algorithms for one-crossing-minor-free graphs. Our matching-mimicking networks could also be applied in finding sequential perfect matching algorithms for the same class of graphs. However, in order to obtain a speedup with this method, we need a subroutine for fast perfect matching in planar graphs. It is known that perfect matching in bipartite planar graphs can be solved in near-linear-time, by a reduction to flow with multiple sources and multiple sinks [5]. Are there similarly fast algorithms for non-bipartite planar perfect matching?

- To what extent can our methods be extended from perfect matching to maximum matching? Datta et al [9] have reduced the search problem to the decision problem for NC planar maximum matching, but it is still not known whether this problem is in NC.

\section{Acknowledgements}

The research of David Eppstein was supported in part by NSF grants CCF-1618301 and CCF-1616248. The research of Vijay Vazirani was supported in part by NSF grant CCF-1815901. We thank Nima Anari for helpful discussions.

\section{REFERENCES}

[1] Nima Anari and Vijay V. Vazirani. 2018. Planar graph perfect matching is in NC. In Proceedings of the 59th Annual IEEE Symposium on Foundations of Computer Science (FOCS), Mikkel Thorup (Ed.). IEEE Computer Society Press, Los Alamitos, California, 650-661. https://doi.org/10.1109/FOCS.2018.00068 arXiv:1709.07822

[2] Nima Anari and Vijay V. Vazirani. 2019. Matching is as easy as the decision problem, in the NC model. Electronic preprint arxiv:1901.10387.

[3] Takao Asano. 1985. An approach to the subgraph homeomorphism problem. Theoretical Computer Science 38, 2-3 (1985), 249-267. https://doi.org/10.1016/ 0304-3975(85)90222-1

[4] Glencora Borradaile, David Eppstein, Amir Nayyeri, and Christian Wulff-Nilsen. 2016. All-pairs minimum cuts in near-linear time for surface-embedded graphs. In Proceedings of the 32nd International Symposium on Computational Geometry (SoCG 2016) (Leibniz International Proceedings in Informatics (LIPIcs)), Sándor Fekete and Anna Lubiw (Eds.), Vol. 51. Schloss Dagstuhl - Leibniz-Zentrum für Informatik, Dagstuhl, Germany, 22:1-22:16. https://doi.org/10.4230/LIPIcs.SoCG. 2016.22 arXiv: 1411.7055

[5] Glencora Borradaile, Philip N. Klein, Shay Mozes, Yahav Nussbaum, and Christian Wulff-Nilsen. 2017. Multiple-source multiple-sink maximum flow in directed planar graphs in near-linear time. SIAM f. Comput. 46, 4 (2017), 1280-1303. https://doi.org/10.1137/15M1042929 arXiv:1105.2228

[6] Erin Wolf Chambers and David Eppstein. 2013. Flows in one-crossing-minorfree graphs. F. Graph Algorithms and Applications 17, 3 (2013), 201-220. https: //doi.org/10.7155/jgaa.00291

[7] S. Chaudhuri, K. V. Subrahmanyam, F. Wagner, and C. D. Zaroliagis. 2000. Computing mimicking networks. Algorithmica 26, 1 (2000), 31-49. https: //doi.org/10.1007/s004539910003 Graph algorithms and applications (Dagstuhl, 1996).

[8] Laszlo Csanky. 1976. Fast parallel matrix inversion algorithms. SIAM 7. Comput. 5, 4 (1976), 618-623. https://doi.org/10.1137/0205040

[9] Samir Datta, Raghav Kulkarni, Ashish Kumar, and Anish Mukherjee. 2018. Planar maximum matching: Towards a parallel algorithm. In Proceedings of the 29th International Symposium on Algorithms and Computation, ISAAC 2018, December 16-19, 2018, Jiaoxi, Yilan, Taiwan (Leibniz International Proceedings in Informatics (LIPIcs)), Wen-Lian Hsu, Der-Tsai Lee, and Chung-Shou Liao (Eds.), Vol. 123. Schloss Dagstuhl - Leibniz-Zentrum für Informatik, Dagstuhl, Germany, 21:121:13. https://doi.org/10.4230/LIPIcs.ISAAC.2018.21

[10] Christian A. Duncan, David Eppstein, Michael T. Goodrich, Stephen G. Kobourov, and Martin Nöllenburg. 2013. Drawing trees with perfect angular resolution and polynomial area. Discrete Comput. Geom. 49, 2 (2013), 157-182. https: //doi.org/10.1007/s00454-012-9472-y

[11] Michael Elberfeld, Andreas Jakoby, and Till Tantau. 2010. Logspace versions of the theorems of Bodlaender and Courcelle. In Proceedings of the 51st IEEE Annual Symposium on Foundations of Computer Science (FOCS 2010). IEEE Computer Society Press, Los Alamitos, California, 143-152. https://doi.org/10.1109/FOCS. 2010.21

[12] David Eppstein and Michael T. Goodrich. 2011. Succinct greedy geometric routing using hyperbolic geometry. IEEE Transactions on Computing 60, 11 (2011), 15711580. https://doi.org/10.1109/TC.2010.257

[13] David Eppstein and Bruce Reed. 2019. Finding maximal sets of laminar 3separators in planar graphs in linear time. In Proceedings of the 30th ACM-SIAM Symposium on Discrete Algorithms (SODA 2019). Society for Industrial and Applied Mathematics, Philadelphia, Pennsylvania, 589-605. https://doi.org/10.1137/ 1.9781611975482 .37

[14] Stephen Fenner, Rohit Gurjar, and Thomas Thierauf. 2019. A deterministic parallel algorithm for bipartite perfect matching. Commun. ACM 62, 3 (2019), 109-115. https://doi.org/10.1145/3306208

[15] Johannes Fischer, Dominik Köppl, and Florian Kurpicz. 2016. On the benefit of merging suffix array intervals for parallel pattern matching. In Proceedings of the 27th Annual Symposium on Combinatorial Pattern Matching, CPM 2016, June 27-29, 2016, Tel Aviv, Israel (Leibniz International Proceedings in Informatics (LIPIcs)), Roberto Grossi and Moshe Lewenstein (Eds.), Vol. 54. Schloss Dagstuhl - Leibniz-Zentrum für Informatik, Dagstuhl, Germany, 26:1-26:11. https://doi. org/10.4230/LIPIcs.CPM.2016.26

[16] Shafi Goldwasser and Ofer Grossman. 2017. Bipartite perfect matching in pseudodeterministic RNC. In Proceedings of the 44th International Colloquium on Automata, Languages, and Programming, ICALP 2017, fuly 10-14, 2017, Warsaw, Poland (Leibniz International Proceedings in Informatics (LIPIcs)), Ioannis Chatzigiannakis, Piotr Indyk, Fabian Kuhn, and Anca Muscholl (Eds.), Vol. 80. Schloss Dagstuhl - Leibniz-Zentrum für Informatik, Dagstuhl, Germany, 87:1-87:13. https://doi.org/10.4230/LIPIcs.ICALP.2017.87

[17] Rohit Gurjar and Thomas Thierauf. 2017. Linear matroid intersection is in quasiNC. In Proceedings of the 49th Annual ACM SIGACT Symposium on Theory of Computing. ACM, New York, 821-830. https://doi.org/10.1145/3055399.3055440 
[18] Rohit Gurjar, Thomas Thierauf, and Nisheeth K. Vishnoi. 2018. Isolating a vertex via lattices: Polytopes with totally unimodular faces. In Proceedings of the 45th International Colloquium on Automata, Languages, and Programming (ICALP 2018) (Leibniz International Proceedings in Informatics (LIPIcs)), Ioannis Chatzigiannakis, Christos Kaklamanis, Dániel Marx, and Donald Sannella (Eds.), Vol. 107. Schloss Dagstuhl - Leibniz-Zentrum für Informatik, Dagstuhl, Germany, 74:1-74:14 https://doi.org/10.4230/LIPIcs.ICALP.2018.74 arXiv:1708.02222

[19] Torben Hagerup, Jyrki Katajainen, Naomi Nishimura, and Prabhakar Ragde 1998. Characterizing multiterminal flow networks and computing flows in networks of small treewidth. 7. Comput. System Sci. 57, 3 (1998), 366-375. https: //doi.org/10.1006/jcss.1998.1592

[20] Dov Harel and Robert E. Tarjan. 1984. Fast algorithms for finding nearest common ancestors. SIAM 7. Comput. 13, 2 (1984), 338-355. https://doi.org/10.1137/0213024

[21] Joseph Ja'Ja' and Janos Simon. 1982. Parallel algorithms in graph theory: planarity testing. SIAM 7. Comput. 11, 2 (1982), 314-328. https://doi.org/10.1137/0211024

[22] Donald B. Johnson. 1987. Parallel algorithms for minimum cuts and maximum flows in planar networks. F. ACM 34, 4 (1987), 950-967. https://doi.org/10.1145/ 31846.31849

[23] Richard M. Karp, Eli Upfal, and Avi Wigderson. 1985. Are search and decision programs computationally equivalent?. In Proceedings of the 17th Annual ACM Symposium on Theory of Computing (STOC 1985). ACM, New York, 464-475. https://doi.org/10.1145/22145.22197

[24] Richard M. Karp, Eli Upfal, and Avi Wigderson. 1986. Constructing a perfect matching is in random NC. Combinatorica 6, 1 (1986), 35-48. https://doi.org/10. 1007/BF02579407

[25] P. W. Kasteleyn. 1967. Graph theory and crystal physics. In Graph Theory and Theoretical Physics, Frank Harary (Ed.). Academic Press, London, 43-110.

[26] Arindam Khan and Prasad Raghavendra. 2014. On mimicking networks representing minimum terminal cuts. Inform. Process. Lett. 114, 7 (2014), 365-371. https://doi.org/10.1016/j.ipl.2014.02.011

[27] Robert Krauthgamer and Inbal Rika. 2012. Mimicking networks and succinct representations of terminal cuts. In Proceedings of the 24th Annual ACM-SIAM Symposium on Discrete Algorithms (SODA '12). Society for Industrial and Applied Mathematics, Philadelphia, Pennsylvania, 1789-1799. https://doi.org/10.1137/1. 9781611973105.128

[28] Charles H. C. Little. 1974. An extension of Kasteleyn's method of enumerating the 1 -factors of planar graphs. In Combinatorial Mathematics (Proc. Second Australian Conf., Univ. Melbourne, Melbourne, 1973) (Lecture Notes in Mathematics), Vol. 403. Springer, Berlin, 63-72. https://doi.org/10.1007/BFb0057377

[29] László Lovász. 1979. On determinants, matchings, and random algorithms. In Fundamentals of computation theory (Proc. Conf. Algebraic, Arith. and Categorical Methods in Comput. Theory, Berlin/Wendisch-Rietz, 1979). Math. Res., Vol. 2. Akademie-Verlag, Berlin, 565-574.
[30] Meena Mahajan and Kasturi R. Varadarajan. 2000. A new NC-algorithm for finding a perfect matching in bipartite planar and small genus graphs. In Proceedings of the Thirty-second Annual ACM Symposium on Theory of Computing. ACM, New York, 351-357. https://doi.org/10.1145/335305.335346

[31] Gary L. Miller and Joseph Naor. 1995. Flow in planar graphs with multiple sources and sinks. SIAM F. Comput. 24, 5 (1995), 1002-1017. https://doi.org/10.1137/ S0097539789162997

[32] Ketan Mulmuley, Umesh V. Vazirani, and Vijay V. Vazirani. 1987. Matching is as easy as matrix inversion. Combinatorica 7, 1 (1987), 105-113. https: //doi.org/10.1007/BF02579206

[33] Bruce Reed and Zhentao Li. 2008. Optimization and recognition for $K_{5}$-minor free graphs in linear time. In LATIN 2008: Theoretical Informatics, 8th Latin American Symposium, Búzios, Brazil, April 7-11, 2008, Proceedings (Lecture Notes in Computer Science), Eduardo Sany Laber, Claudson Bornstein, Loana Tito Nogueira, and Luerbio Faria (Eds.), Vol. 4957. Springer, Berlin, 206-215. https://doi.org/10.1007/ 978-3-540-78773-0 18

[34] Neil Robertson and Paul Seymour. 1993. Excluding a graph with one crossing. In Graph structure theory (Seattle, WA, 1991). Contemp. Math., Vol. 147. America Mathematical Society, Providence, RI, 669-675. https://doi.org/10.1090/conm/ 147/01206

[35] Piotr Sankowski. 2018. NC algorithms for weighted planar perfect matching and related problems. In Proceedings of the 45th International Colloquium on Automata, Languages, and Programming (ICALP 2018) (Leibniz International Proceedings in Informatics (LIPIcs)), Ioannis Chatzigiannakis, Christos Kaklamanis, Dániel Marx, and Donald Sannella (Eds.), Vol. 107. Schloss Dagstuhl - Leibniz-Zentrum für Informatik, Dagstuhl, Germany, 97:1-97:16. https://doi.org/10.4230/LIPIcs.ICALP. 2018.97

[36] Daniel D. Sleator and Robert E. Tarjan. 1983. A data structure for dynamic trees. $\mathcal{F}$. Comput. System Sci. 26, 3 (1983), 362-391. https://doi.org/10.1016/0022-0000(83) 90006-5

[37] Ola Svensson and Jakub Tarnawski. 2017. The matching problem in general graphs is in quasi-NC. In Proceedings of the 58th IEEE Annual Symposium on Foundations of Computer Science (FOCS 2017), Chris Umans (Ed.). IEEE Computer Society Press, Los Alamitos, California, 696-707. https://doi.org/10.1109/FOCS. 2017.70 arXiv:1704.01929

[38] Robert E. Tarjan and Uzi Vishkin. 1985. An efficient parallel biconnectivity algorithm. SIAM 7. Comput. 14, 4 (1985), 862-874. https://doi.org/10.1137/0214061

[39] Vijay V. Vazirani. 1989. NC algorithms for computing the number of perfect matchings in $K_{3,3}$-free graphs and related problems. Information and Computation 80, 2 (1989), 152-164. https://doi.org/10.1016/0890-5401(89)90017-5 (a preliminary version of this paper appeared in Proc. First Scandinavian Workshop on Algorithm Theory (1988), 233-242) 